\title{
Go to the astroparticle physics school with the Toledo Metro Station Totem-Telescope for cosmic rays
}

\author{
Carla Aramo, ${ }^{1, a}$, M. Ambrosio ${ }^{a}$, A. Candelab ${ }^{\text {, P. Mastroserio }}{ }^{a}$ \\ ${ }^{a} I N F N$, Sezione di Napoli Complesso Universitario di Monte S. Angelo, Via Cinthia, Napoli, Italy \\ ${ }^{b}$ Gran Sasso National Laboratory of INFN, Assergi (Aq), Italy \\ E-mail: aramoena.infn.it
}

\begin{abstract}
Among the scientific divulgation activities of the National Institute of Nuclear Physics of Naples (INFN-NA), the installation of the underground cosmic ray telescope at the Toledo Metro Station in Naples in 2014 had a major impact on territory. The detector, consisting of $10 \mathrm{x}-\mathrm{y}$ scintillator planes, read by SiPM, was developed by the Gran Sasso National Laboratory (LNGS), and installed together INFN-NA researchers and the Physics Department of "Federico II" University of Naples (DIPFISUNINA), in collaboration with the Azienda Napoletana Mobilità (ANM). The telescope allows the observation of the muons of the atmospheric cosmic ray showers below 40 meters of depth in the Station through the LEDs light that indicates the trajectory of the particles. At the end of September 2016, as part of the European researchers' night, the telescope was upgraded with a multimedia touch screen Totem that provides telescope's data acquisition via a serial connection, on line data reconstruction and muon track visualization on its wide screen. Moreover, visitors can select videos on cosmic ray physics, as well as on the activities of INFN and of other project partners. The initiative sees engaged INFN-NA, LNGS, DIPFIS-UNINA, ANM and Rotary International. An important aspect is the real-time analysis of Totem's telescope data, which allows the public to see the tracks of the muons. The accumulated data, transmitted to the Web site of INFN-NA, are accessible to the students for educational purposes. A competition was launched at High Secondary Schools, sponsored by the Ufficio Scolastico Regionale (USR), with the aim of engaging teachers and students in astroparticle physics projects. The students have had exposed their own elaborates as posters, computer presentations, artefacts and didactic experiments, during the 4 days of "Futuro Remoto" (May 25-28, 2017). The Totem and its connection to the Toledo telescope open new perspectives for communication and dissemination of scientific culture through the technique of learning by doing: the students realize and show their works done also through the School-Work Alternation (SWA) program.
\end{abstract}

The European Physical Society Conference on High Energy Physics

5-12 July, 2017

Venice

\footnotetext{
${ }^{1}$ Speaker

(C) Copyright owned by the author(s) under the terms of the Creative Commons 


\section{Introduction}

Since May 2014, inside the "Toledo" Metro Station in Naples (Italy), a telescope [1] developed by the Gran Sasso National Laboratory and INFN-NA is present, aimed at detecting the muons of cosmic rays at about 40 meters of depth (Fig. 1 - left). The muon telescope was originally designed for didactic and illustrative purpose. At the end of September 2016, as part of the European researchers' night, it was upgraded with a multimedia touch screen Totem (Fig. 1 right), which provides videos on cosmic ray physics, as well as on the activities of INFN and other project partners through multimedia links. The Totem provides telescope's data acquisition via a serial connection, on line data reconstruction and the muon track visualization on its graphics screen making visible the invisible cosmic radiation penetrating in the rock down to the station. It currently works for communication and dissemination of science culture in Naples and its surroundings. The success of this installation has been very important for our outreach activity. A competition "A scuola di Astroparticelle" [2] was launched in 2016 by INFN at High Secondary Schools, aimed at engaging teachers and students in astroparticle physics projects, directly related to cosmic rays or to other connected items. A large number of students participated to such competition, preparing posters, multimedia presentations, artefacts and even didactic experiments. Results were illustrated through posters and presented to public in May 2017 in Piazza Plebiscito, Naples, during the renowned science festival named "Futuro Remoto", with a considerable success [3]. The first three winners of the competition, plus a class with "honoured mention" [4] had the opportunity to visit the INFN Frascati National Laboratory (LNF) in September 2017.
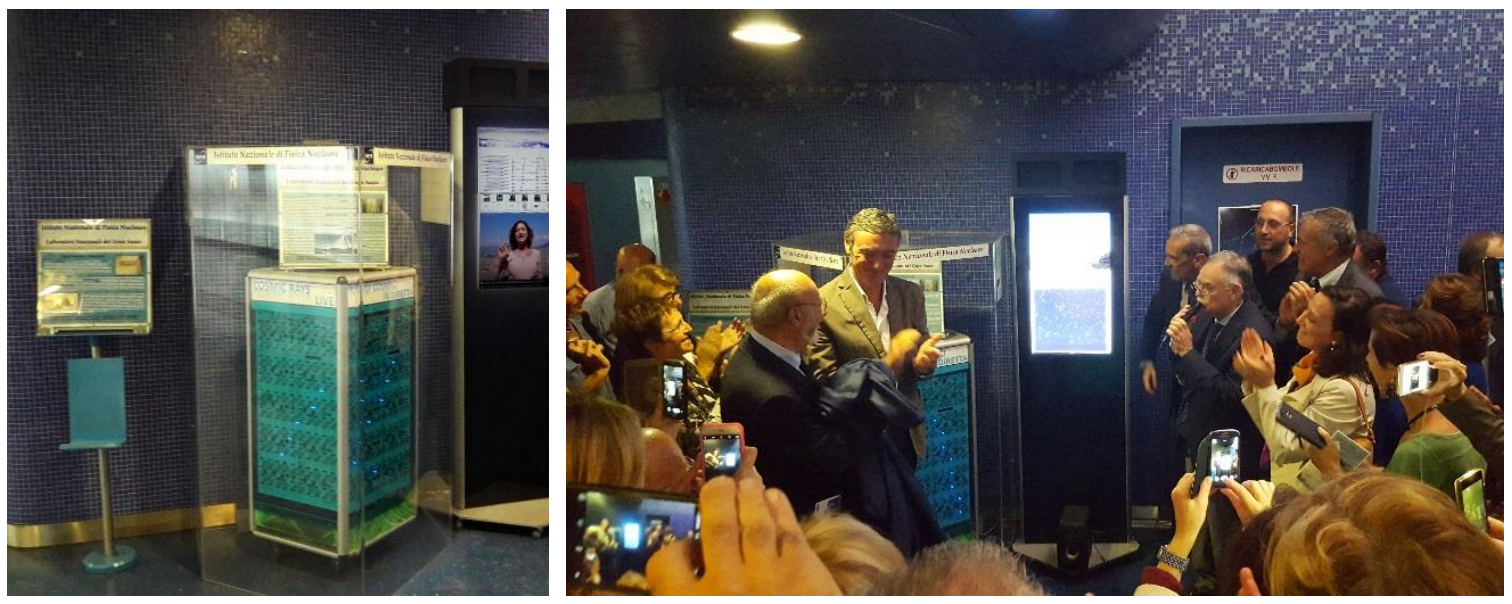

Fig. 1: Muon telescope installed at the Toledo Metro Station - left; multimedia Totem inauguration in September 2016 - right.

\section{The telescope layout and data analysis}

The muon tracking system is composed of 10 double plane horizontal scintillation levels orthogonal each other. Each plane consists of ten solid scintillator strips of $4 \times 1 \times 40 \mathrm{~cm}^{3}$ size with a wavelength-shifter fiber inside carrying the light produced by interaction with a penetrating particle at one end of strip where a Silicon PhotoMultiplier detector (SiPM) collects it generating an electrical signal firing a blue LED (Fig. 2). The scintillator bars are made of doped polystyrene 
and produce blue light. The fiber used is a $1 \mathrm{~mm}$ thick BCF-91A from Saint-Gobaim that absorbs the blue light emitted by the bar and emits green light read by SiPM. The system is composed of 200 electronic channels organized into 10 couples of orthogonal planes allowing the 3D reconstruction of crossing muons. Two front-end boards have been designed to provide voltage bias and readout the SiPMs enclosed in the system to monitor the working parameters and connect the detector to the Totem computer. A controller board unit permits to select different levels of trigger and allows data acquisition for off-line analyses. Data are transmitted on-line to the INFNNA server and stored in a dedicated area where students can access and download them in their personal computers. A data rate of about 4 muons/minute has been obtained under a trigger condition of almost six planes fired in the central part of the telescope. The system is weekly monitored as well strips and SiPM diagnostics.
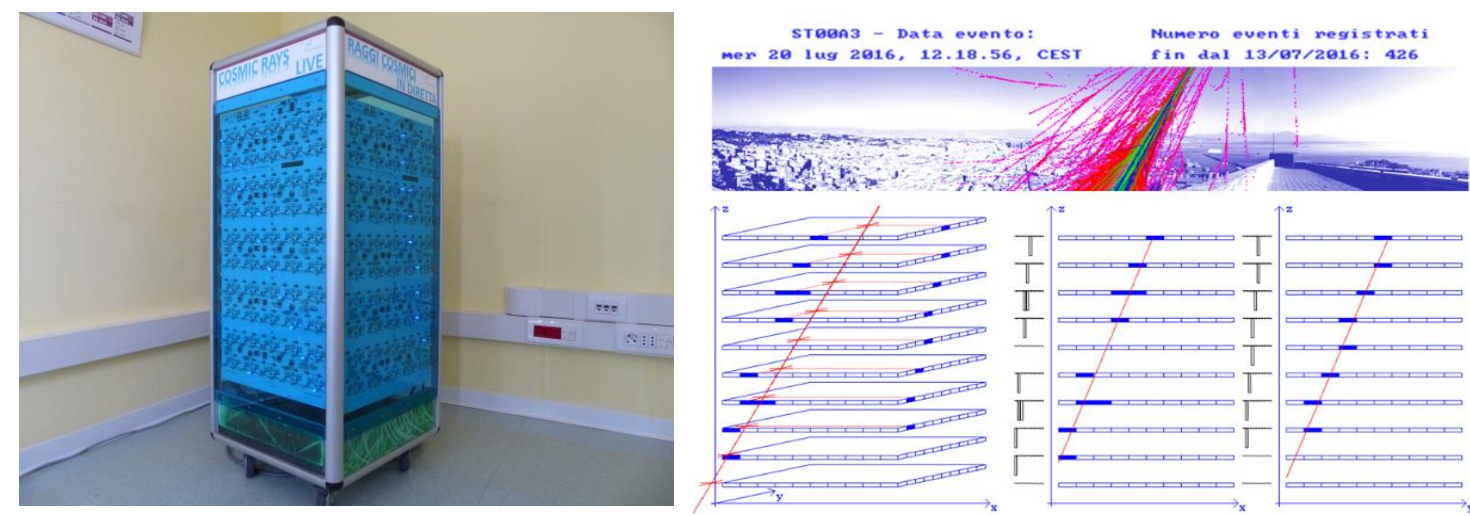

Fig. 2: LED tracks view in the telescope - left; muon tracks as reconstructed by on-line software -right.

Each detected event is reconstructed immediately and its track is plotted on the Totem touch screen. In this way people observing the telescope see the succession of LEDs reproduced on the screen and the invisible particle tracked as in Fig. 2 - left.

In the same time, data are transferred to the INFN-NA server via an internet connection and stored in a database accessible to the students. Each run lasts a day, from time 0.00 to 23.59 . The text files are formatted and named with the data day. Two kind of files are stored. The first contains telescope data as acquired with the serial connection (Fig. 3 - left).

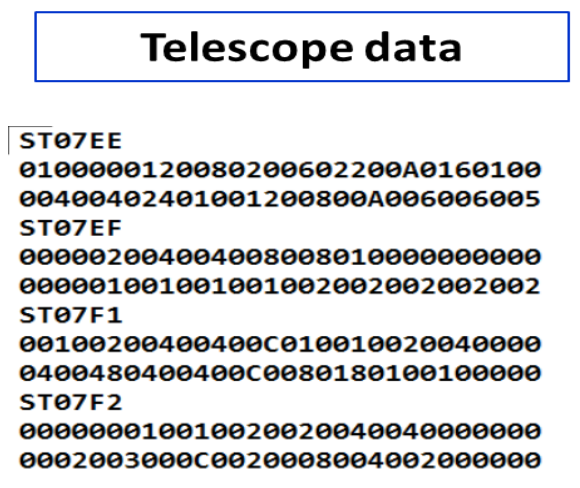

\begin{tabular}{lccc} 
N. ev. & Hour & $\theta$ Angle & $\phi$ Angle \\
\hline ST07EE & $10: 46: 58$ & 18.7 & -23.05 \\
ST07EF & $10: 47: 21$ & -7.24 & -18.31 \\
ST07F1 & $10: 48: 33$ & -14.77 & -22.21 \\
ST07F2 & $10: 48: 57$ & 39.98 & -15.95 \\
ST07F3 & $10: 49: 41$ & 26.56 & -10.79 \\
ST07F4 & $10: 50: 05$ & 26.56 & -37.67 \\
ST07F5 & $10: 50: 23$ & -25.69 & -37.67 \\
ST07F6 & $10: 50: 52$ & -25.69 & -33.77 \\
ST07F7 & $10: 52: 10$ & 26.85 & 20.1 \\
ST07F8 & $10: 53: 05$ & -42.18 & 25.33 \\
ST07F9 & $10: 53: 29$ & -45.46 & -35.18 \\
ST07FA & $10: 53: 53$ & 28.14 & 41.24 \\
ST0805 & $11: 02: 18$ & 7.84 & -13.4 \\
ST0806 & $11: 02: 42$ & 40.1 & -12.88 \\
ST0807 & $11: 03: 06$ & 21.77 & 25.05
\end{tabular}

Fig.3: first kind data file, raw data events -left; second kind data file, reconstruction of the muon tracks right. 
The second records of this file report the strip pattern of all a hundred strips of master side (y view); three hexadecimal bits codify the ten strips of an entire plane with the addition of two dummy strips at the beginning of sequence (three hexadecimal numbers correspond to twelve bit). The third record corresponds to the slave view (x view). By decoding the second and the third records fully event reconstruction is obtained, as shown in Fig. 2 - left.

For each day run, a second file is stored on the server to help the students' work. It contains a simple data reconstruction: for each event it reports the event number, the event time and its inclination in terms of $\theta$ (the $\mathrm{x}-\mathrm{z}$ ) and $\phi$ (the $\mathrm{y}-\mathrm{z}$ ) track angle (Fig. 3 - right).

This second file permits a simplest data management and easy plotting of event frequency, direction and map of the surrounding rock. The Telescope data are at the link http://people.na.infn.it/ totem/Eventi/.

\section{The school competition and School-Work Alternation program}

A competition was launched in November 2016 for High Secondary Schools by INFN, Physics Department of "Federico II" University of Naples, ANM - Azienda Napoletana Mobilità and Rotary International, the Totem donor, and sponsored by the Campania Ufficio Scolastico Regionale (USR), with the aim of engaging teachers and students in astroparticle physics studies and projects.

The subject of the competition was the study of one of the topics of INFN physics and the preparing of posters, multimedia presentations, artifacts and even didactic experiments to be shown for a public exhibition at the end of school year. The students themselves explain to the visitors the problem faced by using the illustrations in their posters and multimedia.

Moreover, seminars on selected topics and visits to the Naples INFN laboratories help students to go deep in to issue also with the help of an INFN tutor guide. Telescope data are the common ground on which to introduce astroparticle issues, but more general problems such as natural radioactivity, relativity, and theories about the origins of the Universe are not excluded. Some works involved the analysis of telescope data and its counting stability too. Not a few were experimental works or suggestions to improve data acquisition.

Nineteen high schools attended the competition, some with more student groups, and twenty-six works were submitted. In each school, introductory seminars were held about the subjects chosen by the students, and other seminars were organized at the request of the schools themselves. Moreover, all the 500 involved students visited the telescope in the Toledo station to see the reconstructed cosmic radiation on the Telescope-Totem and to observe the videos illustrating the research activities supported by the INFN.

Seven of the nineteen schools participating in the competition, also started with INFN-NA a SWA program related to the physics topics chosen by students: 140 students divided into nine groups were assisted by INFN tutors.

They were involved in the Toledo-Telescope activities (Fig. 4 - left) with an introductory seminar on astroparticle physics, a course on security, the visit to the laboratories of INFN experiments, in-depth seminars, small experiments or data analysis or training in writing scientific articles, illustrative brochures and communication of results (Fig. 4 - right). 

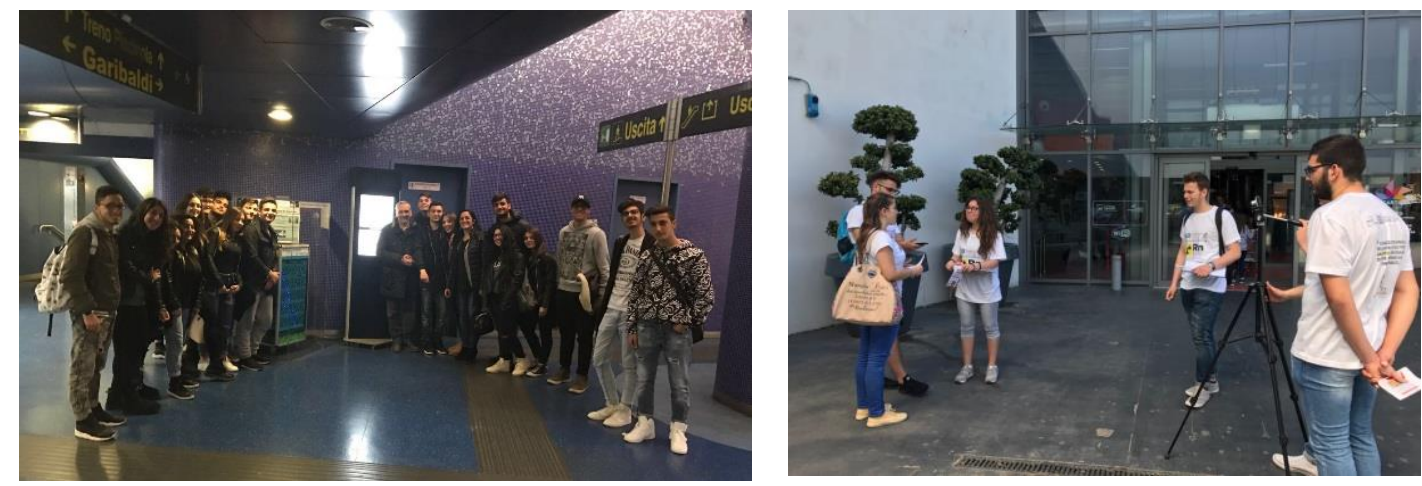

Fig. 4: students visit Toledo-Telescope - left; students in SWA to inform the people about the Radon risks - right.

\subsection{Futuro Remoto}

From May 25 to 28, 2017 in the great Piazza del Plebiscito, Naples, during the renowned science festival "Futuro Remoto" [3] five gazebos were put in place to allow for each day the exhibitions of nine of the twenty-six posters or artefacts done by students (Fig. 5 - left). The event was a great success with about 250,000 visitors and the students, every day, exhibited their works from 10am to $8 \mathrm{pm}$ by helping themselves with the posters they had made. Meanwhile, some researchers in incognito visited the gazebo every day mingling with visitors to select the best jobs and pick out the three winners of the competition.
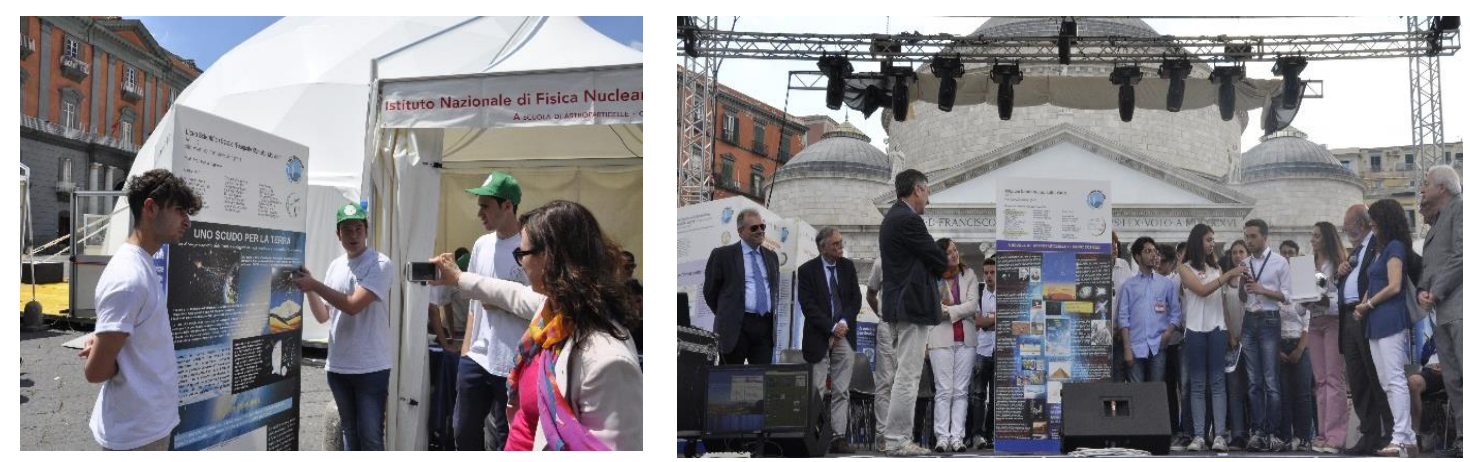

Fig. 5: school exhibits during the four days of "Futuro Remoto" in Piazza del Plebiscito, Naples - left; award ceremony to reward the first three schools - right.

Sunday, May 28, was reserved for the exhibition of the works carried out by the nine working groups of SWA. In the afternoon, the ceremony was held with the participation of INFN President, F. Ferroni and the Director of the INFN-NA G. La Rana. All the posters were aligned on the large Piazza del Plebiscito and the Examination Committee declared the three groups of winning students (Fig. 5 - right) to whom three 8-pack photomultiplier residues of the MACRO experiment at the Gran Sasso Laboratories were awarded. The three winner groups in September had the opportunity of visiting the INFN LNF (Fig. 6 - left).

Moreover, the Liceo Scientifico "A. Nobel” "of Torre del Greco, one of the SWA school, carried its telescope activity in Japan for SKYSEF [5], the free association to bridge School, Science, Culture and Society over the World, held each year in Japan through the Shizuoka Kita High 
School. The work reported an analysis of the angular distribution of muons detected by the Toledo telescope and a muon-radiography of the rock above the telescope itself (Fig. 6 - right).
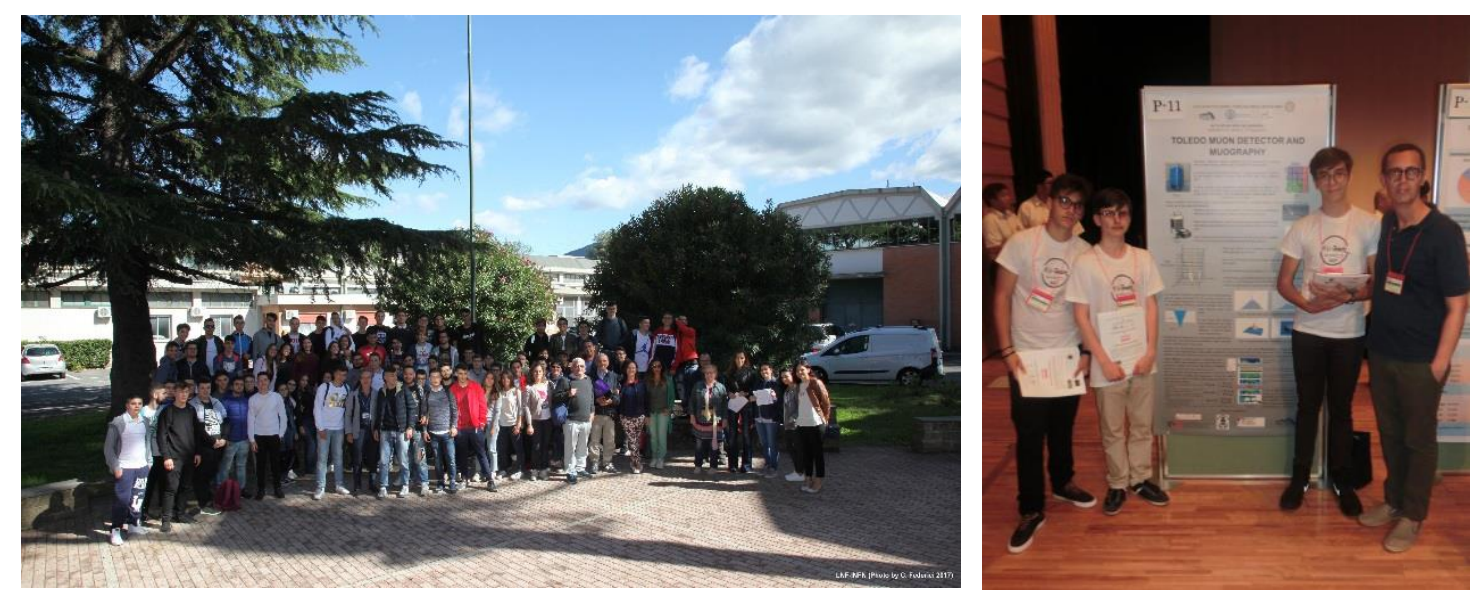

Fig. 6: the winner students during the visit at LNF - left; the students and their teacher at SKYSEF- right.

\section{Conclusion}

The INFN-NA can use for outreach activities an instrument of great educational value (Telescope-Totem) accessible remotely by schools. It enables year-to-year scientific dissemination initiatives involving dozens of schools and hundreds of students. The SWA program realized by INFN-NA in 2016-17 has been highly valued by all participants for its organization and contents. Also the USR-Campania and the City of Science [3] have actively participated in the project and will continue to support it. Not just astroparticle physics but also environmental radioactivity, nano-optics, biosensors, accelerators, theoretical physics, data acquisition techniques, etc. have been studied. The Totem and its connection to the Toledo telescope open new perspectives for communication and dissemination of scientific culture through the technique of learning by doing: the students realize and show their works done also through the SchoolWork Alternation (SWA) program.

\section{Acknowledgment}

The authors would thanks the INFN-NA Director, G. La Rana, for his kind support to this outreach activity, as well as all the researchers involved in the SWA activities. Moreover, the authors are also grateful to S. Arnone and R. Centioni who have organized the visit to LNF for winner students.

\section{References}

[1] F. Arneodo et al., Muon tracking system with Silicon Photomultipliers, NIMA 799 (2015) 166-171

[2] https://www.facebook.com/ascuoladiastroparicelleINFN/

[3] http://www.cittadellascienza.it/futuroremoto/2017

[4] S. Esposito, A demonstration device for cosmic rays telescopes, accepted by Physics Education 2017, arXiv:1708.08677

[5] http://www.scienzaescuola.eu 